\title{
ON BOUNDED CONVOLUTIONS
}

\author{
BY E. R. VAN KAMPEN AND AUREL WINTNER
}

For a type of convolutions of distributions on convex curves, there has been given recently a purely geometrical convergence criterion. $\dagger$ In what follows, this geometrical criterion will be freed from the convexity assumption, thus obtaining in case of bounded spectra a necessary and sufficient convergence criterion which involves only the spectra and not the distribution functions themselves. Only one-dimensional distributions will be considered; it will be clear from the proofs that the transition to multi-dimensional cases is obvious.

If $\beta=\beta(x)$ is a distribution function, let $S(\beta)$ denote its spectrum, and $[S(\beta)]$ the diameter of this spectrum, the diameter $[T]$ of a set $T$ being defined as the least upper bound $(\leqq+\infty)$ of the mutual distances of the pairs of points contained in $T$. The vectorial sum $\ddagger$ of two sets $T_{1}, T_{2}$ will be denoted by $T_{1}(+) T_{2}$, so that $S\left(\beta_{1}{ }^{*} \beta_{2}\right)$ is the closure of $S\left(\beta_{1}\right)(+) S\left(\beta_{2}\right)$; and $S\left(\beta_{1}{ }^{*} \beta_{2}{ }^{*} \cdots\right)$ is the closure of $S\left(\beta_{1}\right)(+) S\left(\beta_{2}\right)(+) \cdots$, whenever the infinite convolution $\beta_{1}{ }^{*} \beta_{2}{ }^{*} \ldots$ is convergent. An infinite convolution $\beta_{1} * \beta_{2} *$. will be called bounded if

$$
\sum_{n=1}^{\infty}\left[S\left(\beta_{n}\right)\right]<+\infty .
$$

It is clear that the boundedness of an infinite convolution is neither necessary nor sufficient for its convergence.

If $\beta_{1}{ }^{*} \beta_{2}{ }^{*} \ldots$ is convergent, its spectrum $S\left(\beta_{1} * \beta_{2}{ }^{*} \ldots\right)$ is a bounded set if and only if $\beta_{1}{ }^{*} \beta_{2}{ }^{*} \ldots$ is a bounded convolution in the sense (1). This is implied by the fact that an infinite convolution $\beta_{1}{ }^{*} \beta_{2}{ }^{*} \ldots$ is bounded if and only if

$$
\left[S\left(\beta_{1}^{*} \cdots \beta_{n}^{*}\right)\right]<\text { const. } \quad(n=1,2, \cdots) .
$$

$\dagger$ E. R. van Kampen and A. Wintner, Convolutions of distributions on convex curves and the Riemann zeta function, American Journal of Mathematics, vol. 59 (1937), pp. 185-186.

¥Cf. B. Jessen and A. Wintner, Distribution functions and the Riemann zeta function, Transactions of this Society, vol. 38 (1935), p. 52 and p. 56. 
The latter statement is clear in the one-dimensional case. In the multidimensional case, where the direction of a maximum diameter can rotate when $n$ varies, the proof follows by projecting these maximum diameters on the axes of a fixed coordinate system and applying then an elementary geometrical argument. The equivalent formulation $\left(1^{\prime}\right)$ of $(1)$ will not be used in what follows.

It will be shown that a bounded infinite convolution $\beta_{1}{ }^{*} \beta_{2}{ }^{*}$ ... is convergent (absolutely convergent) if and only if the series

$$
\sum_{n=1}^{\infty} x_{n}
$$

is convergent (absolutely convergent) for at least one choice of the point $x_{n}$ on $S\left(\beta_{n}\right)$, in which case (2) is uniformly (absolutelyuniformly) convergent for all choices of $x_{n}$ on $S\left(\beta_{n}\right)$.

Those statements of this theorem which concern absolute convergence need not be proved separately, since they are obvious consequences of the corresponding statements concerning convergence.

It is clear from (1) and from the definition of the diameter of a set that if the series (2) is convergent for a single choice of $x_{n}$ on $S\left(\beta_{n}\right)$, then (2) is uniformly convergent for all choices of $x_{n}$ on $S\left(\beta_{n}\right)$. It is similarly seen that (2) is convergent for atl choices of $x_{n}$ on $S\left(\beta_{n}\right)$ if and only if

$$
\sum_{n=1}^{\infty} \mu_{1}\left(\beta_{n}\right) \text { is convergent, }
$$

where

$$
\mu_{m}(\beta)=\int_{-\infty}^{+\infty} x^{m} d \beta(x)
$$

Hence, what one has to prove is that (3) is, in case (1), necessary and sufficient for the convergence of the infinite convolution $\beta_{1} * \beta_{2} * \ldots$. Now it is known $\dagger$ that if

$$
S\left(\beta_{n}\right) \subset K,
$$

† B. Jessen and A. Wintner, loc. cit., p. 57. 
where $K$ is a sufficiently large bounded set independent of $n$, then $\beta_{1}{ }^{*} \beta_{2}{ }^{*} \cdots$ is convergent if and only if both series
(6) $\quad \sum_{n=1}^{\infty} \mu_{1}\left(\beta_{n}\right)$;
$\left(6_{2}\right) \quad \sum_{n=1}^{\infty}\left\{\mu_{2}\left(\beta_{n}\right)-\left(\mu_{1}\left(\beta_{n}\right)\right)^{2}\right\}$

are convergent. Consequently, it is sufficient to prove that

(i) if (1) is convergent, then so is $\left(6_{2}\right)$;

(ii) if $\beta_{1}{ }^{*} \beta_{2}{ }^{*} \ldots$ is convergent and (1) is satisfied, then there exists a bounded set $K$ satisfying (5).

In fact, it clearly follows from (i) and (ii) respectively that (3) is sufficient and necessary for the convergence of $\beta_{1}{ }^{*} \beta_{2}{ }^{*} \ldots$ in case the boundedness condition (1) is satisfied.

Now, it is seen from $(4)$, where $\mu_{0}(\beta)=1$, that the series $\left(6_{2}\right)$ can be written in the form

$$
\sum_{n=1}^{\infty} \int_{-\infty}^{+\infty}\left\{x-\mu_{1}\left(\beta_{n}\right)\right\}^{2} d \beta_{n}(x) \text {, where } \mu_{1}\left(\beta_{n}\right)=\int_{-\infty}^{\infty} x d \beta_{n}(x) .
$$

This series has, in view of the definition of the diameter $\left[S\left(\beta_{n}\right)\right]$ of the spectrum $S\left(\beta_{n}\right)$ of $\beta_{n}$, the majorant

$$
\sum_{n=1}^{\infty}\left[S\left(\beta_{n}\right)\right]^{2},
$$

a series which is convergent in virtue of (1). This proves (i). On the other hand, if $\beta_{1}{ }^{*} \beta_{2}{ }^{*} \ldots$ is convergent, then $\dagger \beta_{n} \rightarrow \lambda$ as $n \rightarrow+\infty$, where $\lambda$ denotes the "unit" distribution function, that is, the distribution function the spectrum of which consists of the single point $x=0$. Hence, if $\beta_{1}{ }^{*} \beta_{2}{ }^{*} \ldots$ is convergent and (1) is satisfied, then there exists for every $\epsilon>0$ an $N=N_{\epsilon}$ such that $S\left(\beta_{n}\right)$ is, for every $n>N$, within the $\epsilon$-vicinity of the point $x=0$, and so (5) holds for a sufficiently large bounded set $K$. This proves (ii).

The Johns Hopkins University

† B. Jessen and A. Wintner, loc. cit., p. 56. 